\title{
The Formation and Destruction of Molecular Clouds and Galactic Star Formation
}

\author{
Shu-ichiro Inutsuka ${ }^{1}$, Tsuyoshi Inoue $^{2}$, Kazunari Iwasaki $^{3}$, \\ Takashi Hosokawa ${ }^{4}$ and Masato I. N. Kobayashi ${ }^{1}$ \\ ${ }^{1}$ Department of Physics, Graduate School of Science, Nagoya University, Nagoya 464-8602, \\ Japan, email: inutsuka@nagoya-u.jp \\ ${ }^{2}$ Division of Theoretical Astronomy, National Astronomical Observatory of Japan, Osawa, \\ Mitaka, Tokyo 181-8588, Japan \\ ${ }^{3}$ Department of Environmental Systems Science, Doshisha University \\ Tatara Miyakodani 1-3, Kyotanabe City, Kyoto 610-0394, Japan \\ ${ }^{4}$ Department of Physics and Research Center for the Early Universe \\ The University of Tokyo, Tokyo 113-0033, Japan
}

\begin{abstract}
We discuss an overall picture of star formation in the Galaxy. Recent high-resolution magneto-hydrodynamical simulations of two-fluid dynamics with cooling/heating and thermal conduction have shown that the formation of molecular clouds requires multiple episodes of supersonic compression. This finding enables us to create a new scenario of molecular cloud formation through interacting shells or bubbles on galactic scales. We estimate the ensembleaveraged growth rate of individual molecular clouds, and predict the associated cloud mass function. This picture naturally explains the accelerated star formation over many million years that was previously reported by stellar age determination in nearby star forming regions. The recent claim of cloud-cloud collisions as a mechanism for forming massive stars and star clusters can be naturally accommodated in this scenario. This explains why massive stars formed in cloud-cloud collisions follows the power-law slope of the mass function of molecular cloud cores repeatedly found in low-mass star forming regions.
\end{abstract}

Keywords. stars: formation - ISM: clouds - ISM: bubbles - ISM: magnetic fields - ISM: structure - ISM: kinematics and dynamics

\section{Introduction}

Recent observational studies of the nearby star-forming regions with the Herschel Space Observatory have convincingly shown a very remarkable fact that stars are born in selfgravitating filaments (e.g., André et al. 2010, 2014) This finding provides a pivot in further theoretical studies of star formation in molecular clouds. The studies of early phases of star formation may set a goal in describing the formation of filamentary clouds where one should explain the apparently universal properties of filamentary clouds such as widths and line-mass of filaments (e.g., Miyama, Narita, \& Hayashi 1987a,b; Nagai, Inutsuka, \& Miyama 1998; Hennebelle \& André 2013). Later phases of star formation correspond to the evolution of filamentary clouds. In contrast to the former, the theoretical study of the latter has been well developed. Indeed, the collapse and fragmentation of filamentary molecular clouds are analyzed in great detail with and without magnetic field (e.g., Nagasawa 1987; Inutsuka \& Miyama 1992, 1997). Those studies have essentially provided possible initial conditions for further protostellar collapse calculations, which are now realistically describing the formation of protostars and protoplanetary disks together with the driving of molecular outflows and/or fast collimated jets (e.g., a review 
by Inutsuka 2012). In this paper we try to explore the former problem, i.e., a theoretical picture for the formation of filamementary molecular clouds.

Before desribing our picture of cloud formation, we point out some implications from the observations of filamentary molecular clouds.

\subsection{Mass Function of Molecular Cloud Cores}

Self-gravitational hydrodynamics is a deterministic process, i.e., the initial conditions and boundary conditions exactly determine the time-evolution of the system. Therefore, the mass function of cores as a result of gravitational fragmentation of a filament should be prescribed essentially by the initial distribution of density fluctuations along the filament. Indeed, Inutsuka (2001) has shown that filamentary clouds with line-mass spectrum $\delta \propto k^{n}$ with $n \sim-1.5$ will produce a mass function of molecular cloud cores whose power law exponent is close to -2.5 , i.e., $d n / d M \propto M^{-2.5}$. Recently Roy et al. (2015) showed that the actual power spectrum of fluctuations along filaments in filamentary molecular clouds of the Gould Belt Survey shows $\delta \propto k^{-1.6}$. If this observed exponent is universal in the sense that similar value is expected in any filamentary molecular cloud, we can attribute the origin of the apparently universal core mass function to the origin of the line-mass fluctuations in filamentary clouds. It is interesting to note that the observed Fourier exponent of line-mass fluctuations is close to the exponent, 5/3, of Kolmogorov Law that are generic property of subsonic tubulence driven by a phase transition (Koyama \& Inutsuka 2002; Audit \& Hennebelle 2005, see the detailed analysis by Hennebelle \& Audit 2007).

\subsection{Formation of Massive Stars in the Filament Paradigm?}

The core mass function observed by André et al. (2010) covers up to $M_{\text {core }} \sim 10 M_{\odot}$. Thus, the success of the filament paradigm explain not only the formation of low mass stars but also the formation of massive stars. This is important in the sense that it means that the formation mechanism of massive stars may be a simple extension of the formation of low mass stars. Since theoretical studies of the formation of massive stars has been controversial for many years (see, e.g., review by Tan et al. 2014), it is crucial to identify direct and clear observational evidence for the formation of massive stars in the filament paradigm. This has been shown by ALMA observation of one of the infrared dark clouds, SDC335.579-0.272, where the actual cloud is composed of multiple filaments of coherent line-of-sight velocities. Peretto et al. (2013) interpreted their observation in terms of multiple filaments falling along their axes towards one of two potential wells which correspond to progenitors of two massive stars. This interpretation explains why the gravitational motions of filamentary clouds naturally realize large mass accretion rates that are supposed to be required for the formation of massive stars.

\section{Formation of Star-Forming Filamentary Clouds}

Recent high-resolution magneto-hydrodynamical simulations of two-fluid dynamics with cooling/heating and thermal conduction have shown that the formation of molecular clouds requires multiple episodes of supersonic compression (Inoue \& Inutsuka 2008, 2009; Heitsch, Stone \& Hartmann 2009). Using three-dimensional MHD simulations including the effects of radiative cooling/heating, chemical reactions, and thermal conductivity, Inoue \& Inutsuka (2012) have investigated the formation of molecular clouds in the magnetized ISM. They have considered the formation of a magnetized molecular cloud due to the accretion of HI clouds formed by the thermal instability. Since the mean density of the initial multi-phase HI medium is an order of magnitude larger than the 

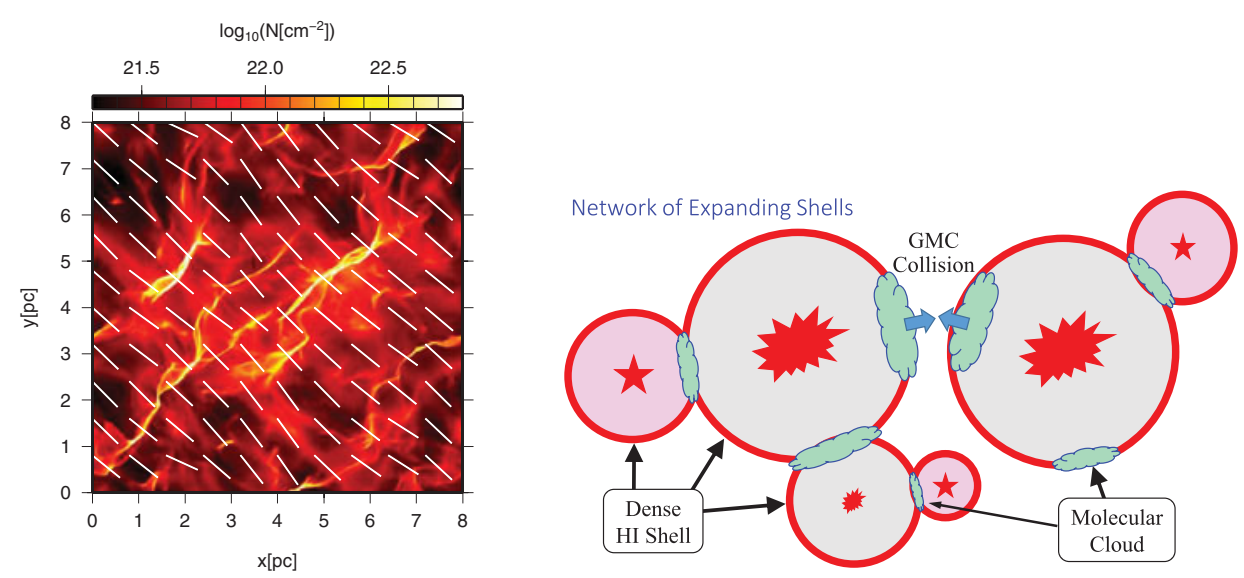

Figure 1. (Left Panel) Face-on column density view of a shock-compressed dense layer in the numerical MHD simulations of Inutsuka et al. (2015). The color scale (in $\mathrm{cm}^{-2}$ ) is shown on the top. The mean magnetic field is in the plane of the layer and its direction is shown by a white bar. Note the formation of dense, magnetized filaments whose axes are almost perpendicular to the mean magnetic field. Fainter "striation"-like filaments can also be seen, which are almost perpendicular to the dense filaments. (Right Panel) A schematic picture of sequential formation of molecular clouds by multiple compressions by overlapping dense shells driven by expanding bubbles. The thick red circles correspond to magnetized dense multi-phase interstellar medium where cold turbulent HI clouds are embedded in warm neutral medium. Molecular clouds can be formed only in the limited regions where the compressional direction is almost parallel to the local mean magnetic field lines or regions experienced excessive number of compressions. An additional compression of a molecular cloud tends to create multiple filamentary molecular clouds that mimics the observation by Herschel Space Observatory. Once the line-mass of a filament exceeds the critical value $\left(\sim 2 C_{\mathrm{s}}^{2} / G\right)$ even in a less massive molecular cloud, star formation starts. In general, star formation in a cloud accelerates with the growth of total mass of the cloud. At limited frequency, giant molecular clouds collide each other. This produces very unstable molecular gas and may trigger very active star formation.

typical diffuse WNM density, the formation timescale is shorter than in the molecular cloud formation through only the accumulation of diffuse WNM. The resulting timescale of molecular cloud formation of $\sim 10 \mathrm{Myr}$ is consistent with the evolutionary timescale of the molecular clouds in the LMC (Kawamura, Mizuno, Minamidani, et al. 2009).

We have done numerical simulations of the additional one-sided compression of the already-formed but faint molecular clouds, and found interesting features of more realistic evolution (Inutsuka et al. 2015). Figure 1 shows a snapshot of the face-on view of a nonuniform molecular cloud compressed by a shock wave travelling at $10 \mathrm{~km} / \mathrm{s}$.

The magnetic field lines are mainly in the dense sheet of the compressed gas. Many dense filaments are created, with axes perpendicular to the mean magnetic field lines. We can also see many faint filamentary structures that mimic observed "striations" and are almost parallel to the mean magnetic field lines. Those faint filaments are feeding gas onto the dense filaments just as envisioned in André et al. (2014).

Although further analysis is required to give a conclusive interpretation of the observations, a simple picture based on repeated compression of a molecular cloud may be a main mechanism for the formation of filamentary molecular clouds.

\section{Bubble Dominated Scenario of Cloud Formation}

HI observation of our galaxy shows many shell-like structures near the Galactic plane (e.g., Hartmann \& Burton 1997; Taylor et al. 2003). Since we identify repeated 
interactions of expanding shock waves as a basic mechanism of molecular cloud formation, we depict the overall scenario of cloud formation in our Galaxy as a schematic picture in Figure 1 (right) (Inutsuka et al. 2015). In this picture, red circles correspond to the remnant of shock waves due to expanding HII regions or old and slowly expanding supernova remnants. Cold HI clouds embedded in warm neutral medium are almost ubiquitously found in the shell of these remnants. Molecular clouds are expected to be formed in special regions where the mean magnetic field is parallel to the direction of shock wave propagation, or where an excessive number of shock wave sweepings happen to be experienced. In this way, molecular clouds can be found only in limited regions on the shells. Note that the typical timescale of each shock wave is on the order of $1 \mathrm{Myr}$, but the formation of a molecular cloud requires many Myr. Some bubbles become invisible as HII regions or supernova remnants many Myrs after their births. Therefore, this schematic picture corresponds to a "very long exposure image" of the real structure of the interstellar medium; Each molecular cloud may acquire a random velocity depending on its location in the most recent bubble that interacts the cloud. Interestingly, this multi-generation picture for evolution of the molecular clouds seems to agree with the observational findings by Dawson et al. $(2011 \mathrm{a}, \mathrm{b})$ who investigated the transition of atomic gas to molecular gas in three clouds in the walls of the Galactic supershells.

In limited circumstances, created molecular clouds collide with one another. This produces very unstable molecular gas and may trigger very active star formation (e.g., Fukui et al.2013, Inoue \& Fukui 2013).

The typical shock velocity of expanding HII regions is $10 \mathrm{~km} / \mathrm{s}$. If the shock velocity of a supernova remnant is much higher than $10 \mathrm{~km} / \mathrm{s}$, the resultant interaction would result in destruction of molecular clouds. The mean velocity of each molecular cloud should be similar or somewhat smaller than the recently swept shock wave. Therefore the velocity dispersion of molecular clouds should be similar or somewhat smaller than $10 \mathrm{~km} / \mathrm{s}$. According to this acquisition process of random velocities, the velocity of a particular cloud is not expected to strongly depend on the mass of the cloud.

\section{Evolution of the Molecular Cloud Mass Function}

In this section, we try to describe the evolution of the mass function of molecular clouds. For that purpose, we first model the growth of molecular clouds. According to Inoue \& Inutsuka $(2009,2012)$, we need multiple episodes of compression of HI clouds to create molecular clouds. The galactic average timescale of each compression should be on the order of $T_{\exp } \sim \mathrm{Myr}$, but can be factor of a few smaller in the spiral arm regions in the Galaxy. Thus, we have to consider the time evolution of cloud mass over a much longer duration than $1 \mathrm{Myr}$.

After each compression, the cloud may slightly expand because of the reduced pressure of the ambient medium, which may cause the loss of the diffuse component of cloud mass. This process has not yet been theoretically studied in detail as far as the authors are aware. Observationally the average column densities of observed molecular clouds do not seem to change very much and always appear to be that corresponding to a visual extinction of several. This means that mass of the cloud is proportional to the cross section of the cloud. Since the compressional formation of molecular material is expected to be proportional to the cross section of the pre-existing cloud, we can model the rate of increase of molecular cloud mass as

$$
\frac{d M}{d t}=\frac{M}{T_{\mathrm{f}}}
$$


where $T_{\mathrm{f}}$ denotes the formation timescale. According to the investigation of molecular cloud formation briefly described in the previous section, Inutsuka et al. (2015) estimate the formation timescale $T_{\mathrm{f}} \sim 10 \mathrm{Myr}$, but this can be factor of a few longer in the interarm regions.

Inutsuka et al. (2015) also estimated the destruction of molecular clouds, by extending the detailed radiation hydrodynamical calculations of the expanding HII region with photodissociative line radiation (Hosokawa \& Inutsuka 2005, 2006a; Hosokawa \& Inutsuka 2006b, 2007) to the cases around a magnetized interstellar medium. For example, they show that a $30 M_{\odot}$ star can destroy a $10^{5} M_{\odot}$ molecular cloud. The mass of the star most responsible for the cloud destruction depends on the actual shape of stellar initial mass function. However, Inutsuka et al. (2015) have shown that the most responsible mass is always $30 \pm 10 M_{\odot}$ for the mass function with a high mass slope $-2.3 \sim-2.7$. This is due to the very sharp increase of the disrupted mass as a function of stellar mass $M_{*}$ for $M_{*}<20 M_{\odot}$, and its saturation for $M_{*}>20 M_{\odot}$.

Suppose that a $10^{5} M_{\odot}$ molecular cloud is eventually disrupted by photodissociating radiation from a $30 M_{\odot}$ star born inside of this cloud, and that star formation is quenched. The condition for this to occur can be written as follows: $10^{5} M_{\odot} \times \epsilon_{\mathrm{SF}}>M_{\text {crit }}\left(30 M_{\odot}\right)$, where $\epsilon_{\mathrm{SF}}$ is the star formation efficiency (the ratio of the total mass of stars to the mass of the parental cloud) and $M_{\text {crit }}\left(30 M_{\odot}\right)$ is the total mass of stars in a group that include only one star whose mass is larger than $30 M_{\odot}$. If the initial mass function is the same as that observed in the solar neiborhood, $M_{\text {crit }}\left(30 M_{\odot}\right)$ should be on the order of $10^{3} M_{\odot}$. If $\epsilon_{\mathrm{SF}}$ is smaller than that satisfies the above inequality, photodissociative disruption is not sufficient and star formation continues with the remaining molecular material in the cloud, this in turn raises $\epsilon_{\mathrm{SF}}$. Thus, we expect that the actual evolution of a molecular cloud finally satisifies the condition $10^{5} M_{\odot} \times \epsilon_{\mathrm{SF}}=M_{\text {crit }}\left(30 M_{\odot}\right)$ when the star formation is eventually quenched. This means that the star formation efficiency should be given by

$$
\epsilon_{\mathrm{SF}}=\frac{M_{\mathrm{crit}}\left(30 M_{\odot}\right)}{10^{5} M_{\odot}} \sim 10^{-2} .
$$

This may explain the reason for the small star formation efficiency in molecular clouds observationally found many decades ago (e.g., Zuckerman \& Evans 1974). For example, according to Hosokawa \& Inutsuka $(2006 \mathrm{~b})$ a $10 M_{\odot}$ star can photodissociate $10^{2} M_{\odot}$ of the surrounding molecular material, but a $10^{2} M_{\odot}$ molecular cloud is not likely to produce a $10 M_{\odot}$ star. Therefore the above argument is not possible with $10 M_{\odot}$ as the mass of the disrupting star.

Now we can estimate the timescale for the destruction of the molecular cloud. The preceding argument has already shown that a $10^{5} M_{\odot}$ molecular cloud can be disrupted within $4 \mathrm{Myr}$. If we assume the initial mass function does not depend on the parental cloud mass, the destruction timescale is not expected to depend on the mass of the cloud larger than $10^{5} M_{\odot}$. This can be understood as follows. The number of dissociating photons is proportional to the number of massive stars, which increases with the mass of the cloud. But the required number of dissociating photons also increases with the mass of the cloud. Therefore the probability of the cloud destruction does not depend on the mass of the cloud, if the shape of the initial mass function does not depend on the mass of the cloud.

The actual disruption timescale $T_{\mathrm{d}}$ should be the sum of the formation timescale of a massive $\operatorname{star}\left(T_{*}\right)$ and the expansion timescale of its HII region, i.e., $T_{\mathrm{d}} \approx T_{*}+4 \mathrm{Myr}$. After one cycle of molecular cloud disruption that takes timescale $T_{\mathrm{d}}$, only a fraction $\epsilon_{\mathrm{SF}}$ of the molecular gas is transformed into stars. Therefore the timescale to completely transform a molecular cloud to stars is $T_{\mathrm{d}} / \epsilon_{\mathrm{SF}} \sim 1.4 \mathrm{Gyr}$. This may explain the so-called "depletion 

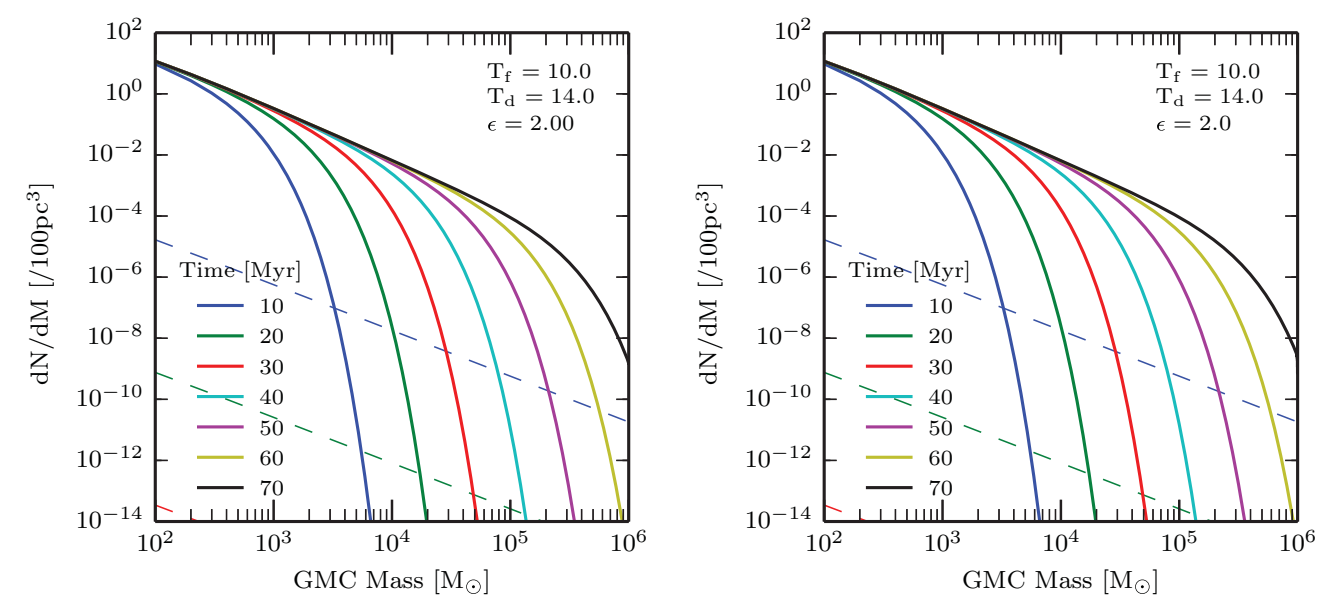

Figure 2. Time evolution of the mass function of molecular clouds. The effect of cloud collisions is not included in the left panel but included in the right panel. The steady state slope of the mass function is essentially determined the ratio of cloud formation and cloud destruction rate as shown in equation (4.6).

timescale" of molecular clouds observationally known as the Schmitt-Kennicutt Law (e.g., Kennicutt 1998).

In order to describe the time evolution of the mass function of molecular clouds over a timescale much larger than Myr (e.g., 10Myr), we adopt coarse graining of short-timescale events of the growth and destruction of clouds, and describe the continuity equation of molecular clouds in mass space.

$$
\frac{\partial N}{\partial t}+\frac{\partial}{\partial M}\left(N \frac{d M}{d t}\right)=-\frac{N}{T_{\mathrm{d}}}+\left(\frac{d N}{d t}\right)_{\text {coll }},
$$

where $N(d M / d t)$ denotes the flux of mass function in mass space, $T_{\mathrm{d}}$ is the cloud disruption timescale, $d M / d t$ describes the growth rate of the molecular cloud as given in Equation (4.1), and the last term on the right-hand side is rate of change of the mass function due to two-body collisions (i.e., cloud-cloud collisions). We assumed the average column density of molecular clouds is always constant $\left(\sim 10^{22} \mathrm{~cm}^{-2}\right)$ and the cross section of a collision can be regarded as a sum of geometrical cross sections of two colliding molecular clouds, and performed time evolution calculations (Kobayashi et al. in prep.). Figure (2) shows typical time evolution of the mass function of molecular clouds. In these figures, we confirm that cloud-cloud collisions do not affect the overall mass function of molecular clouds.

If the contribution from cloud-cloud collisions is negligible, a steady state solution of the above equation is

where

$$
N(M)=\frac{N_{0}}{M_{0}}\left(\frac{M}{M_{0}}\right)^{-\alpha},
$$

$$
\alpha=1+\frac{T_{\mathrm{f}}}{T_{\mathrm{d}}} .
$$

In typical conditions of spiral arm regions, we expect $T_{*} \sim T_{\mathrm{f}}$, and thus, $T_{\mathrm{f}} \lesssim T_{\mathrm{d}}$, which corresponds to $1<\alpha \lesssim 2$. For example, $T_{\mathrm{f}}=10 \mathrm{Myr}$ corresponds to $\alpha \approx 1.7$, which agrees nicely with observations (Solomon et al. 1987; Kramer et al. 1998; Heyer et al. 2001; Roman Duval et al. 2010). However, in quiescent regions with very a limited 
amount of gaseous material away from spiral arms in the Galactic disk, $T_{\mathrm{f}}$ is expected to be large and possibly even larger than $T_{\mathrm{d}}=T_{*}+4 \mathrm{Myr}$. In this case $\alpha$ can be larger than 2. This tendency is actually observed in M33 (Rosolowsky \& Blitz 2005; Gratier et al. 2012) and in M51 (Colombo et al. 2014).

\section{Summary}

In general, dense molecular clouds cannot be created in shock waves propagating in magnetized warm neutral medium without cold HI clouds. In this paper we identify repeated interactions of shock waves in the dense cold HI medium as a basic machanism for creating filametary molecular clouds, which are ubiquitously observed in the nearby interstellar medium. This suggests an expanding-bubble-dominated picture of the formation of molecular clouds in our Galaxy, which enables us to envision an overall picture of the statistics of molecular clouds and resulting star formation. Our conclusions are summarized as follows:

(a) Turbulent cold HI clouds embedded in warm neutral medium can be easily created in the expanding shells of HII regions or the very late phase of supernova remnants. In contrast, the formation of molecular clouds in magnetized ISM need many compression events. One compression corresponds to the order of $1 \mathrm{Myr}$ on average in our Galaxy. The timescale of the cloud formation is a few times $10 \mathrm{Myr}$.

(b) Since the galactic thin disk is occupied by many bubbles, molecular clouds are formed in the overlapping region of (old and new) bubbles. However, since the average lifetime of each bubble is shorter than the timescale of cloud formation, it is difficult to observationally identify the multiple bubbles that create the molecular clouds.

(c) The velocity dispersion of molecular clouds should be originated in the expansion velocities of bubbles. This is estimated to be $\lesssim 10 \mathrm{~km} / \mathrm{s}$ and should not strongly depend on the mass of the molecular cloud.

(d) To describe the growth of molecular cloud mass we can temporally smooth out the evolution over the timescale larger than $\sim 1$ Myr. The resulting mass of each molecular cloud (smoothed over time) is an almost exponentially increasing function of time. Star formation starts even in small molecular clouds, and thus, star formation accelerates on a timescale larger than $\sim 1$ Myr.

(e) Molecular clouds may collide over a timescale of a few times $10 \mathrm{Myr}$, depending on the relative locations in adjacent bubbles. This molecular cloud collision may result in the active star formation in a giant molecular cloud.

(f) The destruction of molecular clouds is mainly due to the dissociative radiation from massive stars of $>20 M_{\odot}$. The probability of cloud destruction is not a sensitive function of the mass of molecular clouds. If the initial mass function does not vary much with the mass of parental molecular clouds, the cloud destruction by $30 M_{\odot}$ stars result in a star formation efficiency on the order of $1 \%$. This property explains the observed constancy of gas depletion rate $\left(\sim[1 \mathrm{Gyr}]^{-1}\right)$ of giant molecular clouds in our and external galaxies, and hence, corresponds to Kennicutt-Schmidt Law.

( $g$ ) The steady state of the evolution of cloud mass function corresponds to the power law with exponent $n$ in the range $1<n \lesssim 2$ in the spiral arm regions of our Galaxy. However, a larger value of the exponent such as $n>2$ is possible in the inter-arm regions.

In many disk galaxies, star forming regions tend to be located in spiral structures, and thus, many bubbles should be located in spiral structures. It might be interesting to study how this bubble dominated picture of the formation of molecular cloud corresponds to the shaping of spiral structure itself. This requires a more comprehensive study of the dependence of the various timescales relevant in this picture, on the ambient ISM density 
and pressure, radiation contents, metallicity, etc. We hope that the present picture will provide a useful step for understanding star formation in the Galactic scale.

\section{References}

André, P., Men'shchikov, A., Bontemps, S. et al. 2010, A\&A, 518, L102

André, P., Di Francesco, J., Ward-Thompson, D. et al. 2014, Protostars and Planets VI, (Univ of Arizona Press) eds. H. Beuther, R. Klessen, C. Dullemond, \& Th. Henning

Audit, E. \& Hennebelle, P. 2005, A\&A , 433, 1

Colombo, D., Hughes, A., Schinnerer, E. et al. 2014, ApJ 784, 3

Dawson, J. R., McClure-Griffiths, N. M., Kawamura, A. et al. 2011, ApJ 728, 127

Dawson, J. R., McClure-Griffiths, N. M., Dickey, J. M. et al. 2011, ApJ 741, 85

Gratier, P., Braine, J., Rodriguez-Fernandez, N. J., et al. 2012, A\&A, 542, A108

Hartmann, D. \& Burton, W. B. 1997, Atlas of Galactic Neutral Hydrogen (New York: Cambridge Univ. Press)

Heyer M. H. et al. 2001, ApJ 551, 2, 852

Heitsch F., Stone J. M., Hartmann L. W., 2009, ApJ, 695, 248

Heitsch F., \& Hartmann L. W. (2014) MNRAS 443, 230

Hennebelle, P. \& André, P. 2013, A\&A , 560, 68

Hennebelle, P. \& Audit, E., 2007, A\&A , 465, 431

Hosokawa, T. \& Inutsuka, S. 2005, ApJ , 623, 917

Hosokawa, T. \& Inutsuka, S. 2006, ApJ , 646, 240

Hosokawa, T. \& Inutsuka, S. 2006, ApJL , 648, L131

Hosokawa, T. \& Inutsuka, S. 2007, ApJ , 664, 363

Inoue, T. \& Inutsuka, S. 2008, ApJ , 687, 303

Inoue, T. \& Inutsuka, S. 2009, ApJ , 704, 161

Inoue, T. \& Inutsuka, S. 2012, ApJ , 759, 35

Inutsuka, S. 2001, ApJ 559, L149

Inutsuka, S. 2012, Prog. Theor. Exp. Phys., Vol. 2012, 01A307

Inutsuka, S. \& Miyama, S. M. 1992, ApJ, 388, 392

Inutsuka, S. \& Miyama, S. M. 1997 ApJ, 480, 681

Inutsuka, S., Inoue, T. Iwasaki, K., \& Hosokawa, T. 2015, A $\& A$ 580, A49

Kawamura, A., Mizuno, Y., Minamidani, T., et al. 2009, ApJS, 184, 1

Koyama, H. \& Inutsuka, S. 2002, ApJL 564, Issue 2, L97

Kramer C. et al. 1998 A\&A 329, 249

Kennicutt, R. C. Jr. 1998, ApJ 498, 541

Miyama, S. M., Narita, S., \& Hayashi, C. 1987, Prog. Theo. Phys. 78, 1051

Miyama, S. M., Narita, S., \& Hayashi, C. 1987, Prog. Theo. Phys. 78, 1273

Nagai, T., Inutsuka, S., \& Miyama, S. M. 1998, ApJ 506, 306

Nagasawa, M. 1987, Prog. Theor. Phys., 77, 635

Peretto, N., Fuller, G. A. Duarte-Cabral, A. et al. 2013, A\& A 555, A112

Roy, A., André, Ph, Arzoumanian, D. et al. 2015, A\&\&A in press (arXiv:1509.01819)

Roman Duval J. et al. 2010 ApJ 723, 492

Rosolowsky, E. \& Blitz, L. 2005, ApJ, 623, 826

Solomon P. M. et al. 1987 ApJ 319, 730

Tan, J. C., Beltrán, M. T., Caselli, P. et al. (2014) Protostars and Planets VI, (Univ of Arizona Press) eds. H. Beuther, R. Klessen, C. Dullemond, \& Th. Henning,

Taylor, A. R., Gibson, S. J., Peracaula, M. et al. 2003, AJ 125, 3145

Zuckerman, B. \& Evans, N. J. 1974, ApJ, 192, L149 